\title{
Biped Robot Modeling and Control Using Controlled Hybrid Automata
}

\author{
Ying Shang \\ Department of Electrical and Computer Engineering, Southern Illinois University Edwardsville, Edwardsville, USA
}

Email address:

yshang@siue.edu

\section{To cite this article:}

Ying Shang. Biped Robot Modeling and Control Using Controlled Hybrid Automata. International Journal of Systems Science and Applied Mathematics. Vol. 2, No. 4, 2017, pp. 75-82. doi: 10.11648/j.ijssam.20170204.11

Received: May 18, 2017; Accepted: May 27, 2017; Published: July 18, 2017

\begin{abstract}
Hybrid systems are dynamical systems consisting of interacting discrete event and continuous state subsystems. A controlled hybrid automaton is a hybrid automaton whose continuous-state dynamics are described by inhomogeneous differential equations. This paper presents a sufficient condition for the existence of global non-terminating solutions in controlled hybrid automata. The condition is based on a recursive algorithm that can always terminate after a finite number of iterations to a limit set of states, i.e. the fixed point of the recursion. If the fixed point is non-empty, then there exists a measurable control under which the hybrid automaton generates a global non-terminating solution. The more important is that this result can also be used to infer the existence of global solutions to compositions of controlled hybrid automata, thereby providing a foundation for the analysis of large scale hybrid systems. The controlled hybrid automata model can be used for robotics system modeling and control. By solving the global non-terminating solution to controlled hybrid automata, the biped robots can be guaranteed to keep the walking gait without falling down.
\end{abstract}

Keywords: Hybrid Automata, Decidability Problem, Backward Reachability

\section{Introduction}

Hybrid systems are dynamical systems consisting of interacting discrete event and continuous state subsystems. The first important hybrid system model was the model proposed by Hans Witsenhausen in 1966 [16], known as the autonomous hybrid systems. Switched systems [2] proposed by Brockett provide a more convenient description than Witsenhausen's model. Branicky's framework [1] is the most general on of the preceding models because it allows continuous control signals in the vector spaces and discrete control systems in the guard condition sets. A controlled hybrid automaton, a subset of Branicky's model, is a hybrid automaton, whose underlying continuous dynamics are modelled by inhomogeneous ordinary differential equations. More advanced hybrid systems were introduced in recent research work, such as stochastic hybrid systems [13].

There are several different problems proposed in the hybrid research area. This section discusses three fundamental analysis problems: the verification problem, the stability problem and the existence problem, respectively. The verification problem asks if a hybrid system design is correct, i.e. if the hybrid system satisfies certain properties. The simplest verification problem is a reachability question, which is to ask whether or not some bad states are reachable from the initial state of the system. The stability problem looks for the conditions under which the equilibrium point of a hybrid system is stable. The existence problem tries to find the conditions under which the solution to a hybrid system exists. Before one verifies if the system's solution satisfies certain conditions, it is important to make sure whether or not the solution exists at all, and if so, whether there are many possible solutions satisfying the system's dynamics. The existence problem, therefore, is the foundation of the verification and stability problems.

The main result of this paper is a sufficient condition for the existence of global non-terminating solutions in controlled hybrid automata. A global non-terminating solution to a controlled hybrid automaton is a hybrid trajectory with an infinite number of switching instants in an infinite time interval. The condition is based on a recursive algorithm that terminates after a finite number of iterations to a set of states. If this set is non-empty, then there exists a measurable control under which the hybrid automaton generates a global non-terminating solution. The more 
important is that this result can also be used to infer the existence of global solutions to compositions of controlled hybrid automata, thereby providing a foundation for the analysis of large scale hybrid systems.

The application of the main results is in biped-robot modeling and control. In recent publications [12, 15] in robotics, it was projected that robotics leads social innovation in the future, moreover, software/hardware initiatives will be main focuses for robotics. The author has previous work in hardware design in robotic arm [3] and mobile robot tracking [10]. This paper provides main contribution in gait control of different types of robots [4, 5, 6, 9, 14]. Especially, in biped robotic applications, the existence of a non-terminating solution would be able to guarantee the robot keep walking without falling down.

This paper is organized as follows: Section 2 introduces the definition of controlled hybrid automata and illustrates the modeling method for a kinematic robotic leg. Section 3 presents the recursive algorithm for the non-terminating solution to controlled hybrid automata. If given a hybrid system in which multiple cycles support non-terminating solutions with known fixed point of the recursive algorithm, then the composition of these cycles will also have nonterminating solutions provided that the fixed points of individual cycles have a non-empty intersection. In Section 4, the compositional result is illustrated in a biped walking robot to ensure the robot keep walking without falling down.

\section{Controlled Hybrid Automata}

A hybrid automaton is a finite state machine with a set of real-valued variables. The state of a hybrid automaton consists of a continuous-valued vector and a discrete-valued vector. The continuous evolution is determined by an ordinary differential equation or a differential inclusion and the discrete evolution is controlled by a logical predicate, called the guard. A hybrid automaton consists of a finite set $X=\left\{x_{1}, x_{2}, \cdots, x_{n}\right\}$ of real-valued variables and a labeled directed graph $(Q, E) . Q$ is a set of vertices and $E$ is a set of directed arcs or edges between vertices. Vertices represent the discrete states or modes of a hybrid automaton. A hybrid state $(q, x)$ consists of a discrete mode $q \in Q$ and a particular value $x$ of the continuous variables in $X$. In each discrete mode, the real-valued continuous variables evolve according to a flow condition described by a differential equation $\dot{x}(t)=f_{i}(x(t), u(t)) \quad$ or $\quad$ a differential inclusion $\dot{x}(t) \in f_{i}(x(t), u(t))$. The hybrid automaton whose flow conditions are in the differential equation forms is called a controlled hybrid automaton. A solution $\chi$ to a hybrid automaton $H$ consists of three components, hybrid time trajectory $\tau$, continuous variable $x$, discrete state $q$. These components satisfy three conditions:

1. Initial condition: $(x(0), q(0)) \in \operatorname{Init}(q)$, where $\operatorname{Init}(q)$ is the initial discrete states;

2. Continuous evolution: in each time interval where a discrete transition does not occur, the continuous variable $x$ satisfies the invariant condition $\operatorname{Inv}(q)$ and the flow condition $\dot{x}(t)=f_{i}(x(t), u(t))$;

3. Discrete evolution: when a discrete transition occurs, the continuous state $x$ satisfies a transition condition $G\left(q, q^{\prime}\right)$, that enables the discrete transitions. If the discrete transition is an autonomous jump, then the continuous state satisfies the reset condition. The reset condition is a function mapping from a direct arc $\left(q_{i}, q_{j}\right)$ to a value of $\Re^{n}$.

A solution $\chi$ sometimes is called an execution to a hybrid automaton.

Example: A one leg walking robot is used to illustrate how to construct hybrid automaton models of physical systems. The leg is parameterized by the variables $q=(x, l, \theta)$ as shown in Figure 1. These variables correspond to the lateral position of the body, the length of the leg and the angular displacement of the leg, respectively.

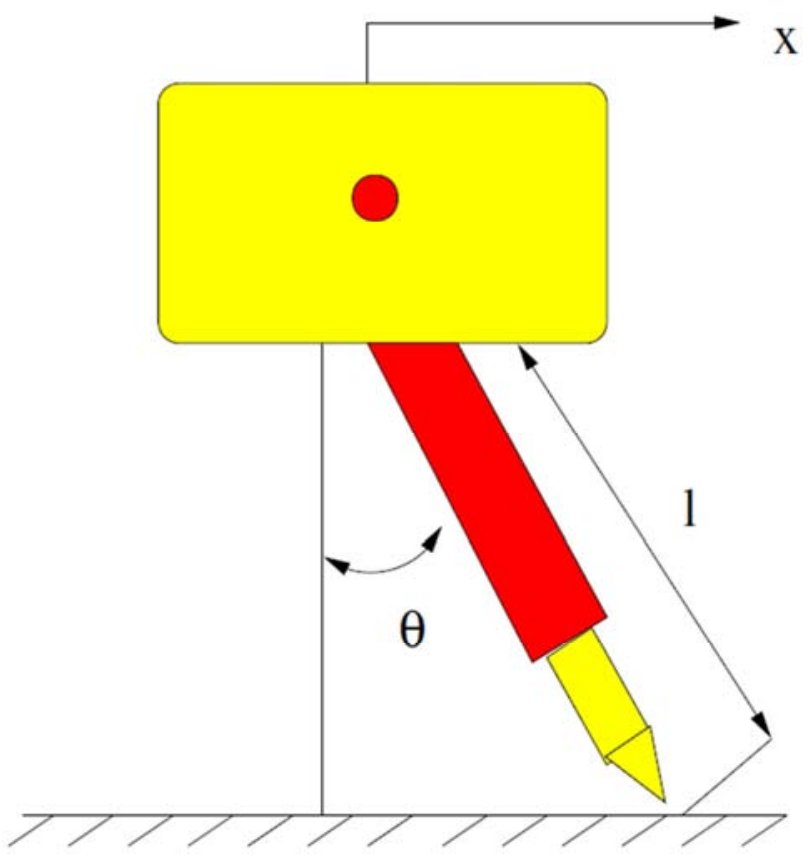

Figure 1. The kinematic leg [8].

Assuming that the height of the body off the ground remains fixed, so when the leg is lifted off the ground, the body will not fall down. Assume the inputs for the system are the velocities $u_{1}=\dot{l}$ and $u_{1}=\dot{\theta}$. When the angular displacement of the leg arrives at the boundary condition $\theta=\theta_{1}$, the length of the leg is shortened by an impulsive input forcing the foot off the ground. The angular position $\theta$ will be controlled to arrive at the boundary $\theta=\theta_{2}$. While this is happening, the leg is lengthened so it lands on the ground when the boundary condition is met. This system may be represented by hybrid automaton in Figure 2, where the discrete state OFF denotes the state when the foot is off the ground, and ON denotes the state when the foot is on the ground. $S_{\{O N, O F F\}}$ and $S_{\{O F F, O N\}}$ are the guards for the transitions between two states. 


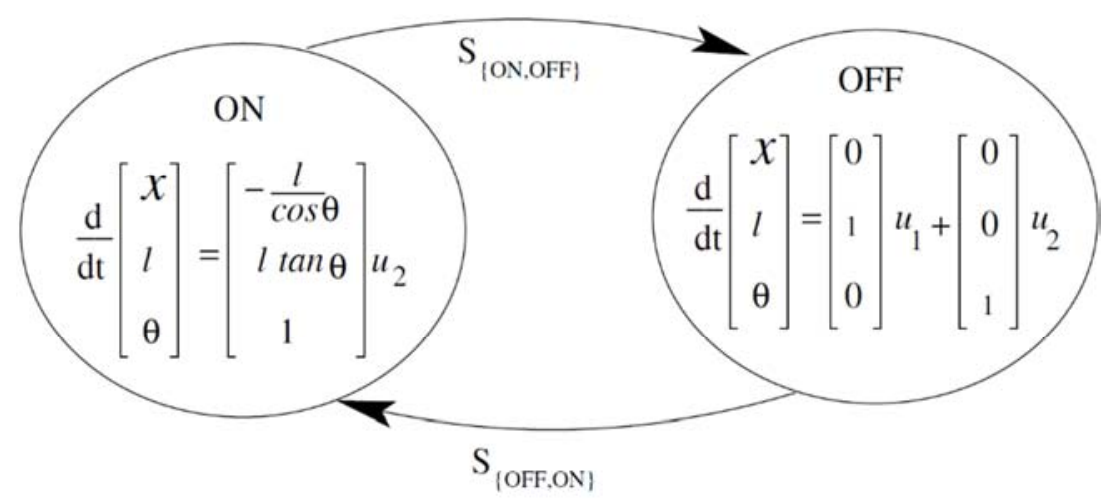

Figure 2. The controlled hybrid automaton model for the kinematic leg.

In this case, the state of kinematic leg on the ground is the set of points

$$
S=\left\{q \in \mathfrak{R}^{3}: l \cos \theta=h\right\}
$$

where $\mathrm{h}$ is the fixed height. The equations of motions are given by

$$
\frac{d}{d t}\left[\begin{array}{l}
x \\
l \\
\theta
\end{array}\right]=\left[\begin{array}{l}
0 \\
1 \\
0
\end{array}\right] u_{1}+\left[\begin{array}{l}
0 \\
0 \\
1
\end{array}\right] u_{2}
$$

when the foot is off the ground and

$$
\frac{d}{d t}\left[\begin{array}{l}
x \\
l \\
\theta
\end{array}\right]=\left[\begin{array}{c}
-\frac{l}{\cos \theta} \\
l \tan \theta \\
1
\end{array}\right] u_{2}
$$

when the foot is in contact with the ground. The guard sets are

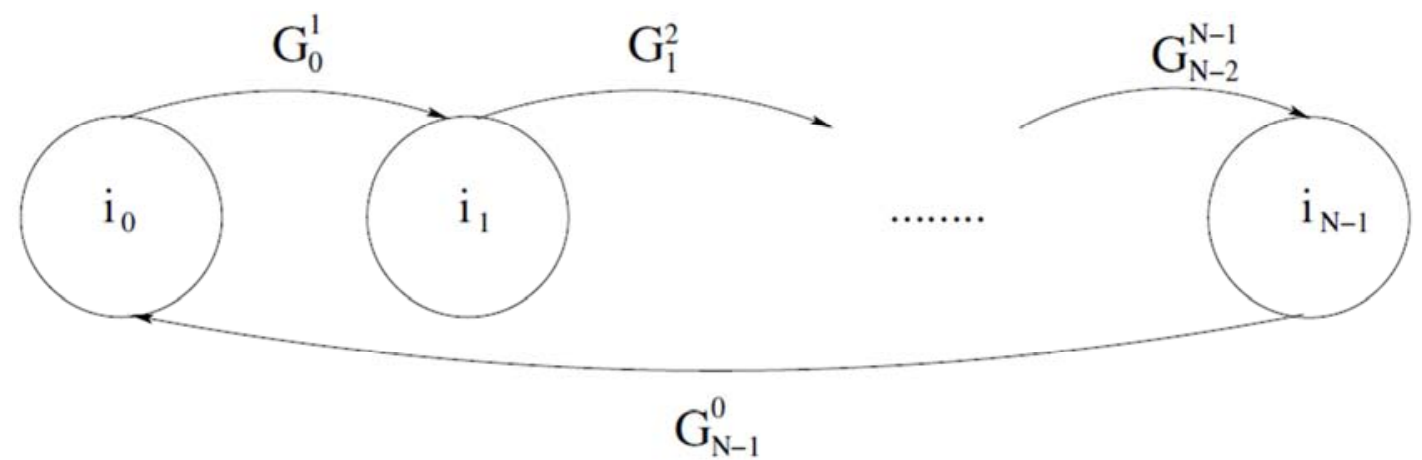

Figure 3. Controlled hybrid automaton.

$\alpha=\left(i_{0}, i_{1}\right),\left(i_{1}, i_{2}\right), \cdots\left(i_{N-1}, i_{N}\right),\left(i_{N}, i_{0}\right)$,

and assume that is logically accepted by the hybrid automaton. We define the recursion by the equations

$$
\left\{\begin{array}{cccc}
\Gamma_{0} & = & G_{0}^{1} \\
\Gamma_{j+1} & = & G_{\bmod (N-j-1)}^{\bmod (N-j)} \bigcap \operatorname{Pre}\left(\Gamma_{j}\right)
\end{array}\right.
$$

$$
\begin{aligned}
& S_{\{O N, O F F\}}=\left\{\left(q=(x, l, \theta) \in \mathfrak{R}^{3}: \theta=\theta_{1}\right\},\right. \\
& S_{\{O F F, O N\}}=\left\{\left(q=(x, l, \theta) \in \mathfrak{R}^{3}: \theta=\theta_{2}\right\} .\right.
\end{aligned}
$$

When the value of $\mathrm{q}$ is in the set of $S_{\{O N, O F F\}}$, the leg will lift off the ground and vice versa. In order to have the robot keeps hopping, we need to have a non-terminating or nonblocking solution between the two discrete states (ON/OFF). Next section, we will present the solution to the nonterminating solution to controlled hybrid automata.

\section{Non-terminating Solutions to Controlled Hybrid Automata}

If we consider a controlled hybrid automaton given in Figure 3, for a set $K$ of $X$, we define the precondition set of $K$ under event $(i, j)$ by the equation Pre $(K)=\bigcap_{x_{f} \in K} \operatorname{Pre}\left(x_{f}\right)$. In fact, the precondition set consists all states $x$ in $\mathrm{X}$ such that any final state $\left(x_{f}, j\right)$ can be reached from $(x, i)$. Given the trace (i.e. loop) of $\mathrm{N}$ events, 
for $\mathrm{j}=0,1, \ldots$

Lemma 1 [11]: If $\Gamma_{N}$ is non-empty, then for any $x_{f} \in G_{0}^{1}$, there exists a $T_{f} \geq 0$ and a trajectory $q \in Q \times X$, such that for any initial state $q_{0} \in Q_{0}, q$ solves the hybrid automaton, $q$ has the trace $\alpha$, and $x\left(T_{f}\right)=x_{f}$.

Lemma 2 [11]: If $x \notin \Gamma_{N}$, then there exists at least on point in $G_{0}^{1}$ that is not reachable from $x_{0}$ under a trajectory $q$ with trace $\alpha$.

Lemma 3 [11]: $\Gamma_{N}=\Gamma_{2 N}$.

Proposition 1: If $\Gamma^{*}$ is non-empty, then there exists a trajectory $q \in Q \times X$, such that for any initial state $q_{0} \in Q_{0}$, $q$ solves the hybrid automaton, $q$ has the trace $\alpha^{*}$, and there exists a sequence of switching instants $\left\{\tau_{j}\right\}_{j=0}^{\infty}$ such that $x\left(\tau_{j}\right) \in \Gamma$.

Proof: from Lemma 3, we know that $\Gamma^{*}=\Gamma_{N}$. Any initial state $q_{0} \in Q_{0}$ can be reached by any point in $G_{0}^{1}$ based on Lemma 2. Therefore, a point in the $\Gamma^{*}$ can be reached along the trajectory $q$, therefore, the trajectory $q$ solves the hybrid automaton with the trace $\alpha$.

Remarks: The recursive algorithm has two advantages:

1. Because the sequence $\left\{\Gamma_{j N}\right\}_{j=0}^{\infty}$ converses to a fixed point $\Gamma^{*}$ after one integration, we can compute the fixed point without more than one cycle to reduce the computational complexity.

2. Moreover, this backward reachability algorithm ensures the compositional analysis of hybrid automata. Composition in this paper means that the fixed point of the two or more individual strings can be used to compute the fixed point of the composed strings as shown in Proposition 2.

Proposition 2: Given a hybrid automaton shown in Figure 4 and let $\alpha$ and $\beta$ be two traces

$$
\begin{aligned}
& \alpha=\left(i_{0}, i_{1}\right),\left(i_{1}, i_{2}\right), \cdots\left(i_{N-1}, i_{0}\right), \\
& \beta=\left(j_{0}, j_{1}\right),\left(j_{1}, j_{2}\right), \cdots\left(i_{M-1}, i_{0}\right),
\end{aligned}
$$

with $i_{0}=j_{0}$. Then the string $\alpha \beta$ is logically accepted by the hybrid automaton and $\Gamma_{\alpha \beta}^{*}=\Gamma_{\alpha}^{*} \cap \Gamma_{\beta}^{*}$.

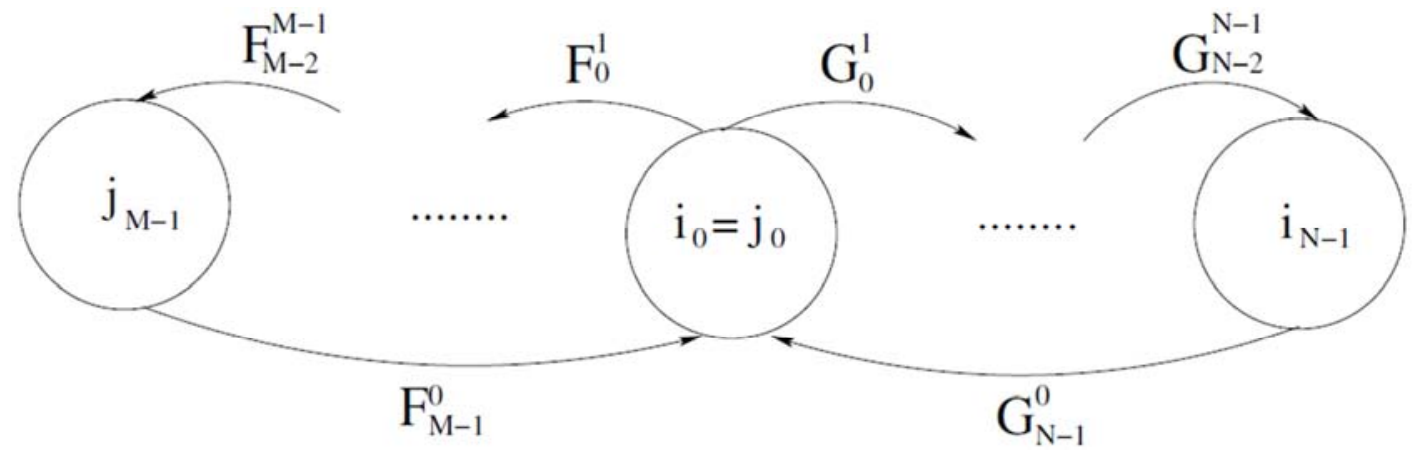

Figure 4. A cascaded controlled hybrid automaton.

Proof: First, the left inclusion $\Gamma_{\alpha \beta}^{*} \supseteq \Gamma_{\alpha}^{*} \cap \Gamma_{\beta}^{*}$ needs to be proven. By the definition of the recursion for trace ${ }_{0}^{\alpha}$ in Eq. (1),

$$
\left\{\begin{array}{ccc}
\Gamma_{0}^{\alpha} & = & G_{0}^{1} \\
\Gamma_{n+1}^{\alpha} & = & G_{\bmod (N-n) 1)}^{\bmod (N-n)} \bigcap \operatorname{Pre}\left(\Gamma_{n}^{\alpha}\right)
\end{array}\right.
$$

For $n=0, \cdots, N-1$. By Lemma 3, we know $\Gamma_{\alpha}^{*}=\Gamma_{N}^{\alpha}$. By the definition of the recursion for trace ${ }_{0}^{\alpha}$ in Eq. (1),

$$
\left\{\begin{array}{ccc}
\Gamma_{0}^{\beta} & = & \Gamma_{N}^{\alpha} \cap F_{0}^{1} \\
\Gamma_{m+1}^{\beta} & = & F_{\bmod (M-m-1)}^{\bmod (M-m)} \cap \operatorname{Pre}\left(\Gamma_{m}^{\beta}\right)
\end{array}\right.
$$

For $m=0, \cdots, M-1$. After the recursive computation, $\Gamma_{N}^{\alpha} \cap \Gamma_{M}^{\beta}=\Gamma_{\alpha \beta}^{*}$, which includes $\Gamma_{\alpha}^{*} \cap \Gamma_{\beta}^{*}$.
Second, the right inclusion $\Gamma_{\alpha \beta}^{*} \subseteq \Gamma_{\alpha}^{*} \cap \Gamma_{\beta}^{*}$ needs to be proven. This inclusion direction is trivial due to the definition of the precondition set. Any point in the fixed point of the trace $\alpha \beta$ will be able to reach to the final state in under the individual trace $\alpha$ and $\beta$. Hence we proved that the equality $\Gamma_{\alpha \beta}^{*}=\Gamma_{\alpha}^{*} \cap \Gamma_{\beta}^{*}$ holds.

In the next section, a biped walking robot is used to illustrate the main contribution of the paper, i.e. the preceding proposition for compositional analysis.

\section{Application to a Biped Walking Robot}

This section uses the biped walking robotic system as an example to illustrate the main results on the existence of nonterminating solutions for controlled hybrid automata. The biped robot in this thesis is modified from the simplest special case of the passive-dynamic models in [3]. Two rigid legs are connected by a frictionless hinge at the hip. A point- 
mass is at the hip and infinitesimal point-masses at the feet.

The walking cartoon of the point-foot model is shown in

Figure 5.

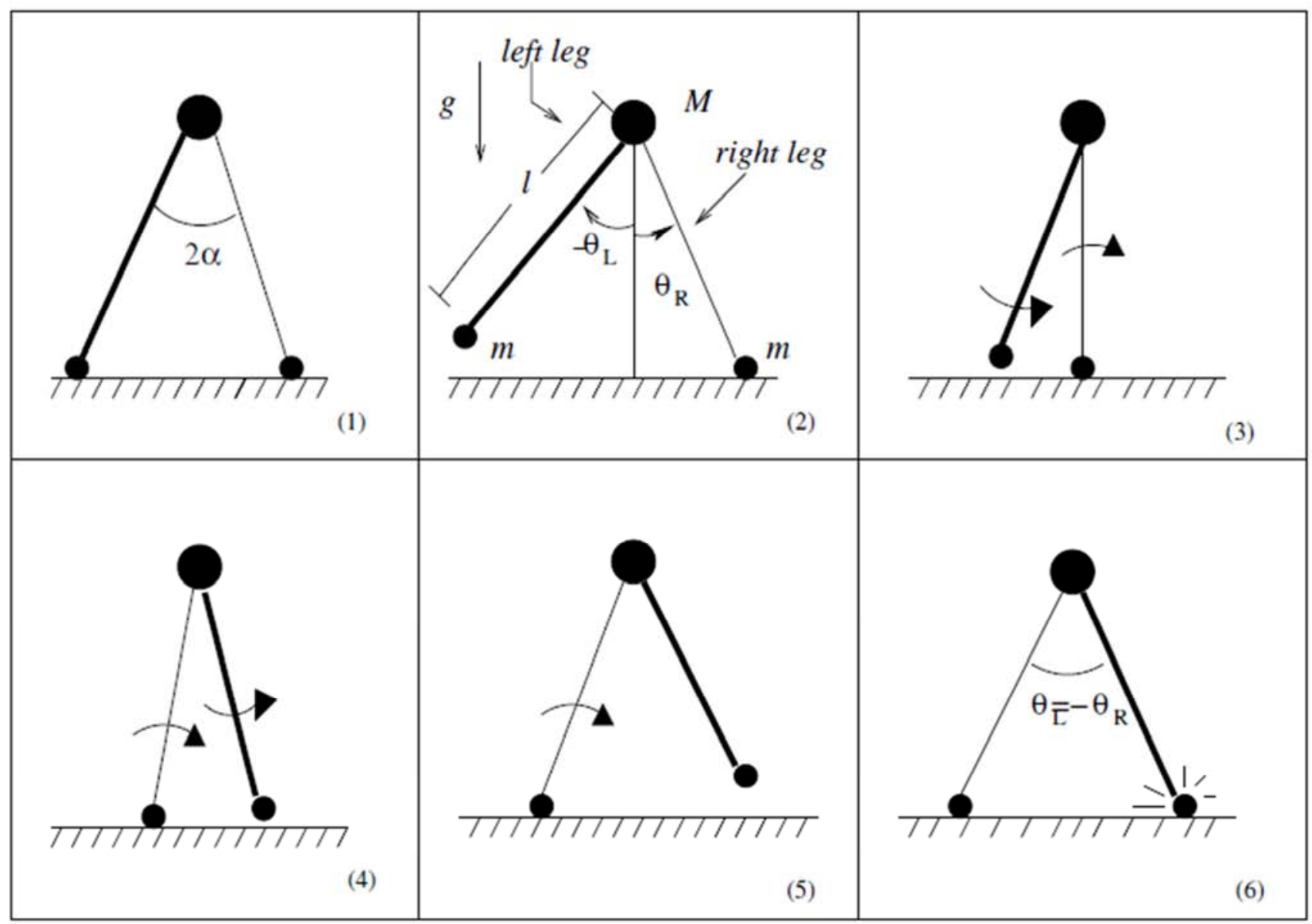

Figure 5. The walking pattern of the rigid-leg biped robot [7].

The walking dynamics of the biped robot contains three modes: the double legs support the body, the right leg supports the body, and the left leg supports the body. Each state is described by a differential equation. The system is parameterized by the $q=(\theta, \dot{\theta})$, where $\theta=\left(\theta_{R}, \theta_{L}\right)$. When the right leg supports the body, the equations of motion are given by

$$
\frac{d}{d t}\left[\begin{array}{c}
q_{1} \\
q_{2} \\
q_{3} \\
q_{4}
\end{array}\right]=\left[\begin{array}{c}
q_{3} \\
q_{4} \\
\sin \left(q_{2}\right) \\
-q_{3}^{2} \sin \left(q_{1}-q_{2}\right)+\cos \left(q_{1}\right) \sin \left(q_{1}-q_{2}\right)
\end{array}\right]
$$

which are derived from the nonlinear inverted pendulum equations

$$
\frac{d}{d t}\left[\begin{array}{c}
\ddot{\theta}_{R} \\
\ddot{\theta}_{L}
\end{array}\right]=\left[\begin{array}{c}
\sin \theta_{R} \\
-\dot{\theta}_{R}{ }^{2} \sin \left(\theta_{R}-\theta_{L}\right)-\cos \theta_{R} \sin \left(\theta_{R}-\theta_{L}\right)
\end{array}\right] .
$$

Similarly, when the left leg supports the body, the equations of the motion are given by

$$
\frac{d}{d t}\left[\begin{array}{c}
q_{1} \\
q_{2} \\
q_{3} \\
q_{4}
\end{array}\right]=\left[\begin{array}{c}
q_{3} \\
q_{4} \\
-q_{4}^{2} \sin \left(q_{2}-q_{1}\right)+\cos \left(q_{2}\right) \sin \left(q_{2}-q_{1}\right) \\
\sin \left(q_{1}\right)
\end{array}\right],
$$

which are derived from the nonlinear inverted pendulum equations

$$
\frac{d}{d t}\left[\begin{array}{c}
\ddot{\theta}_{L} \\
\ddot{\theta}_{R}
\end{array}\right]=\left[\begin{array}{c}
\sin \theta_{L} \\
-\dot{\theta}_{L}^{2} \sin \left(\theta_{L}-\theta_{R}\right)-\cos \theta_{L} \sin \left(\theta_{L}-\theta_{R}\right)
\end{array}\right] .
$$

When the double legs support the body, the equations of the motion are given by

$$
\frac{d}{d t}\left[\begin{array}{l}
q_{1} \\
q_{2}
\end{array}\right]=\left[\begin{array}{l}
u_{1} \\
u_{2}
\end{array}\right]
$$

The walking robot dynamic can be modelled as a controlled hybrid automaton shown in Figure 6. 


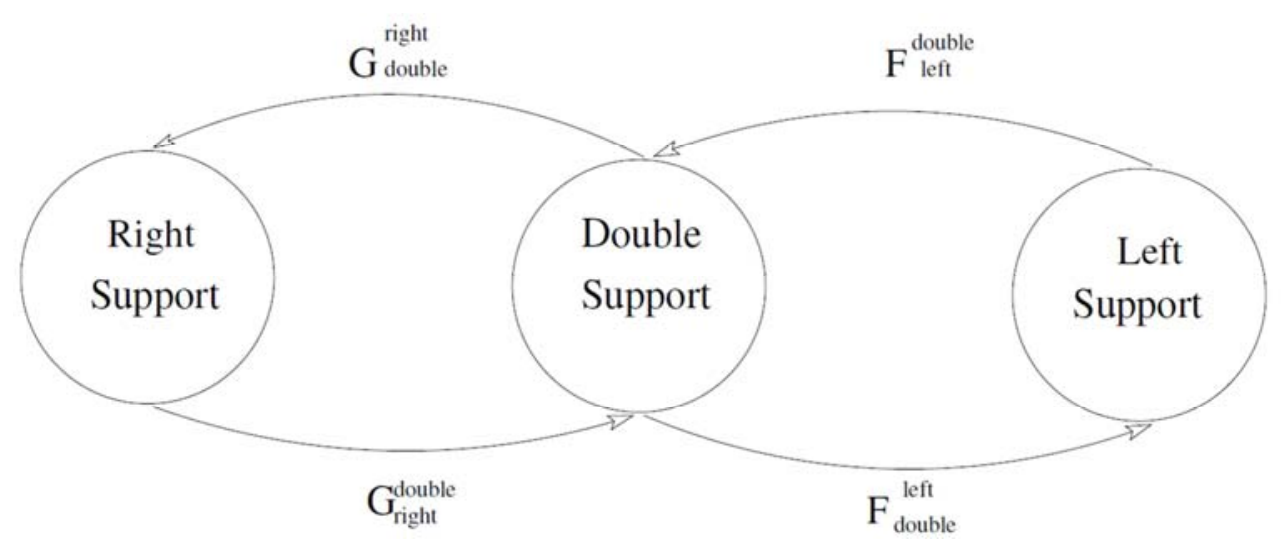

Figure 6. Controlled hybrid automaton model for the biped walking robot.

When the right leg supports the body, the equations of motion are given by. After mathematical derivation, the minimum symmetric walking period is obtained as $\tau=3.8121$. The guard conditions are given as follows:

$$
\begin{aligned}
& G_{\text {double }}^{\text {right }}=\left\{q \mid q_{1}\left(k_{1} \tau\right)=-q_{2}\left(k_{1} \tau\right), q_{3}\left(k_{1} \tau\right)=q_{4}\left(k_{1} \tau\right)=\frac{1+e^{\tau}}{1-e^{\tau}} q_{1}(k \tau)\right\} \\
& G_{\text {right }}^{\text {double }}=\left\{q \mid q_{1}\left(k_{1} \tau\right)=-q_{2}\left(k_{1} \tau\right), k_{1} \in \mathrm{Z}\right\}
\end{aligned}
$$

and

$$
\begin{aligned}
& F_{\text {double }}^{\text {left }}=\left\{q \mid q_{1}\left(k_{2} \tau\right)=-q_{2}\left(k_{2} \tau\right), q_{3}\left(k_{2} \tau\right)=q_{4}\left(k_{2} \tau\right)=\frac{1+e^{\tau}}{1-e^{\tau}} q_{1}\left(k_{2} \tau\right)\right\} \\
& F_{\text {left }}^{\text {double }}=\left\{q \mid q_{1}\left(k_{2} \tau\right)=-q_{2}\left(k_{2} \tau\right), k_{2} \in \mathrm{Z}\right\} .
\end{aligned}
$$

If defining the trace $\alpha$ as that the state variables change from the double support mode to the right support mode and then back to the double support mode. This means that the right leg is always the support leg and the left leg is the swing leg. The walking gait is the left leg swings forward and backward and the right leg supports the body. Similarly, the trace $\beta$ is that the state variables change from the double support mode to the left support mode and then back to the double support mode. This walking gait is that the right leg swings forward and backward and the left leg supports the body.

If defining the biped robot walking gait as trace $\alpha \beta$ which means either the right leg or the left leg becomes a support leg and the other leg is the swing leg. The backward recursion is used to compute the fixed points of traces $\alpha, \beta$ and $\alpha \beta$ respectively. It is not hard to find that $\Gamma_{\alpha}^{*}=G_{\text {double }}^{\text {right }}, \Gamma_{\beta}^{*}=F_{\text {double }}^{\text {right }}$, and

$$
\begin{aligned}
& \Gamma_{\alpha \beta}^{*}=\left\{q \mid q_{1}\left(k_{1} \tau\right)=-q_{2}\left(k_{1} \tau\right), q_{3}\left(k_{1} \tau\right)=q_{4}\left(k_{1} \tau\right)=\frac{1+e^{\tau}}{1-e^{\tau}} q_{1}\left(k_{1} \tau\right),\right. \\
& \left.q_{3}\left(k_{2} \tau\right)=q_{4}\left(k_{2} \tau\right)=\frac{1+e^{\tau}}{1-e^{\tau}} q_{1}\left(k_{21} \tau\right), k_{1}=2 n, k_{2}=2 n+1, n \in Z\right\} \\
& \bigcup\left\{q \mid q_{1}\left(k_{1} \tau\right)=-q_{2}\left(k_{1} \tau\right), q_{3}\left(k_{1} \tau\right)=q_{4}\left(k_{1} \tau\right)=\frac{1+e^{\tau}}{1-e^{\tau}} q_{1}\left(k_{1} \tau\right),\right. \\
& \left.q_{3}\left(k_{2} \tau\right)=q_{4}\left(k_{2} \tau\right)=\frac{1+e^{\tau}}{1-e^{\tau}} q_{1}\left(k_{21} \tau\right), k_{1}=2 n+1, k_{2}=2 n, n \in Z\right\} .
\end{aligned}
$$

This illustrates the main result in Proposition 2, the intersection of $\Gamma_{\alpha}^{*} \cap \Gamma_{\beta}^{*}$ is the same as $\Gamma_{\alpha \beta}^{*}$, which implies that the biped walking gat can be analyzed by two independent walking gaits (left to ground, and right to ground), respectively. The angle vs. time, angular velocity vs. time, and phase portraits during the four walking steps are shown in Figures 7-9, and the one step of the biped walking robot is shown in Figure 10.

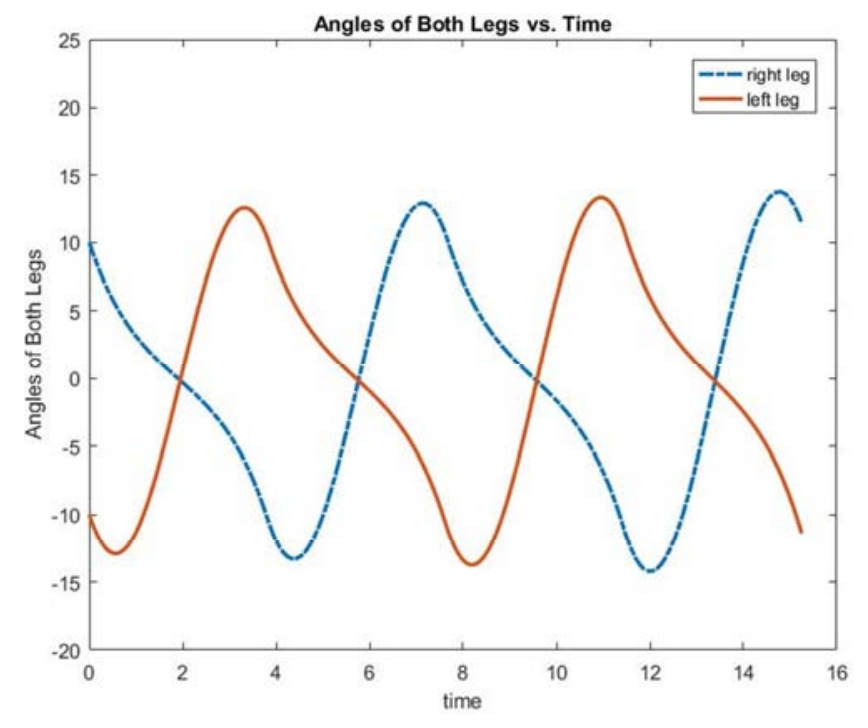

Figure 7. Angles vs. time for the biped robot. 


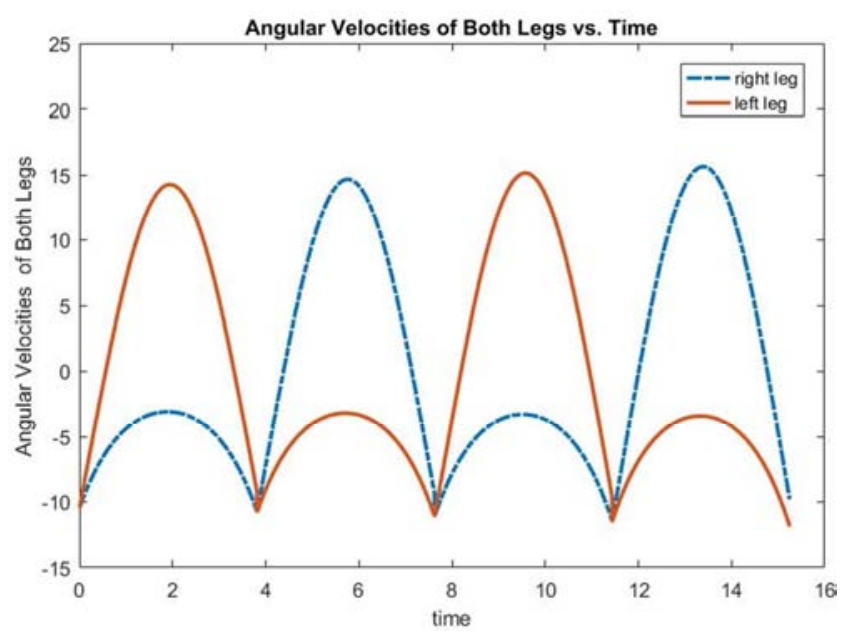

Figure 8. Angular velocities vs. time for the biped robot.

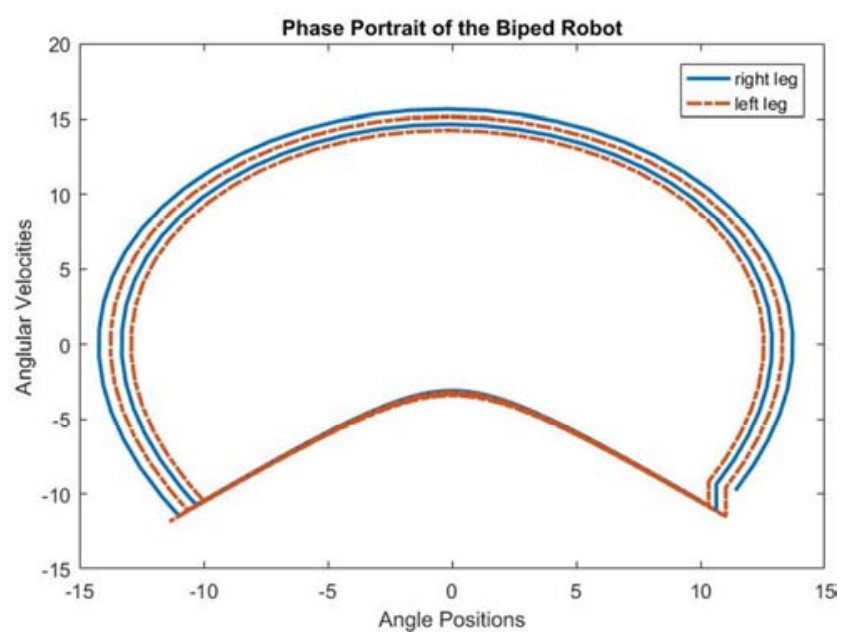

Figure 9. Phase portrait of the walking biped robot.

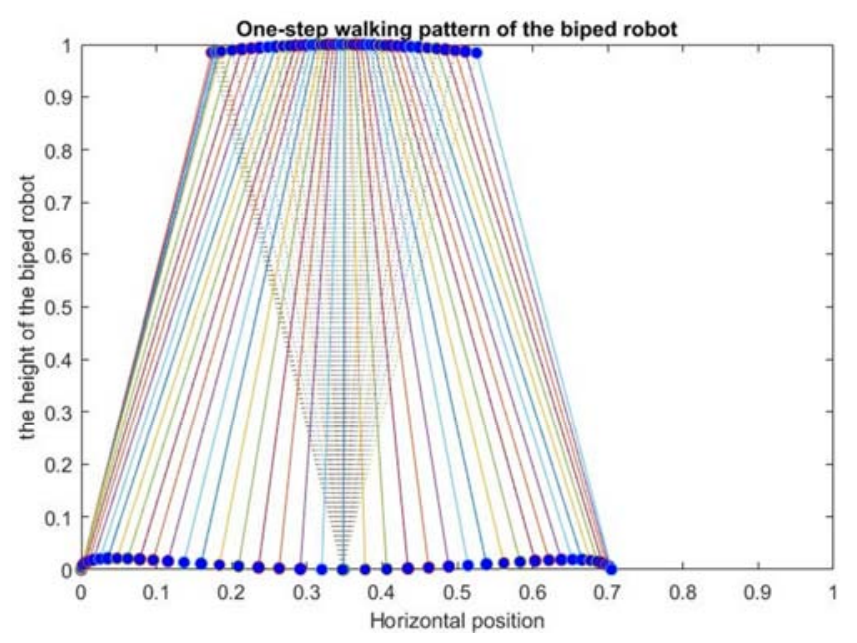

Figure 10. The biped robot one step walking gait animation.

\section{Conclusion}

The main result of this thesis is a sufficient condition for the existence of nonterminating solutions to a controlled hybrid automaton. The sufficient condition is based on a recursive algorithm that computes an inner approximation of the backward reachability sets. In this paper, the algorithm guarantees to terminate in a finite number of iterations. If the fixed-point of the recursive algorithm is non-empty, then we know there exists a control supporting the specified cycle. If we are given a hybrid system in which multiple cycles support non-terminating solutions with known fixed points, then the composition of these cycles will also have nonterminating solutions provided that the fixed points have a non-empty intersection. In this paper, the compositional result is illustrated in a biped walking robot to ensure the robot keep walking without falling down. Controller synthesis and optimal controller design are potential future research directions.

\section{References}

[1] M. S. Branicky, Wivek S. Borkar, S. K. Mitter, "A unified framework for hybrid control," IEEE Trans. on Automatic Control, Vol. 43, Issue 1, pp. 31-45, 1998.

[2] R. W. Brockett, "Hybrid models for motion control systems," Essays in Control, pp. 29-53, Birkhauser, Boston, 1993.

[3] K. E. Colthier, Y. Shang, “ A geometric approach for robotic arm kinematics wit hardware design, electrical design, and implementation. Journal of Robotics, 2010.

[4] S. Cubero, Editor, Industrial Robotics: Theory, Modeling and Control, Pro Literatur Verlag, Germany, 2016.

[5] A. R. C. Donati, et. al. "Long-term training with a Brainmachine interface-based gait protocol induces partial neurological recovery in paraplegic patients," Scientific Report, 6: 30383, 2016.

[6] L. A. Fuente, M. A. Lones, A. P. Turner, L. S. Caves, S. Stepney, A. M. Tyrrell. "Adaptive robotic gait control using coupled artificial signaling networks, Hopf oscillators and inverse kinematics. IEEE Congress on Evolutionary Computation (CEC), June 20-23, 2013.

[7] M. Garcia, A. Chattejee, A. Ruina, and M. Coleman, "The simplest walking model: stability, complexity, and scaling," ASME J. Biomech. Eng. Vol. 120, pp. 281-288, 1998.

[8] B. Goodwine, J. W. Burdick, "Controllability of kinematic control systems on stratified configuration spaces," IEEE Trans. on Automatic Control, Vol. 46, No. 3, pp. 358-368, 2001.

[9] K. Leibrandt, C. Bergeles, G-Z. Yang, "Concentric tube robots: rapid, stable path-planning and guidance for surgical use", IEEE Robotics \& Automation Magazine, Vol: PP, Issue: 99, May 2017, DOI: 10.1109/MRA.2017.2680546.

[10] Y. K. Leow, Y. Shang, "Mobile robot tracking in wireless sensor networks", Computer in Educaion Journal, Vol. 1, Issue 1, pp. 36-44, 2010.

[11] Y. Shang, "The existence of non-terminating solutions to controlled hybrid automata," master thesis, University of Notre Dame, 2002.

[12] S. Tadokoro, "Robotics leads social innovation without boarders for the future of humanity," IEEE Robotics \&Automation Magazine, Vol. 24, Issue 1, 4-4, 2017. 
[13] A. Teel, J. Hespanha, "Stochastic hybrid systems: a modeling and stability theory tutorial" Proc. of the $54^{\text {th }}$ Conference on Decision and Control, Dec. 15-18, 2015.

[14] T. Umedachi, T. Kano, A Ishiguro, B. A. Trimmer, "Gait control in a soft robot by sensing interactions with the environment using self-deformation," Royal Society Open Science, 3: 160766, 2016.
[15] B. Vanderborght, "The impact of hardware and open-source initiatives on robotics," IEEE Robotics \&Automation Magazine, Vol. 24, Issue 1, 4-4, 2017.

[16] H. S. Witsenhausen, "A class of hybrid-state continuous-time dynamic systems," IEEE Trans. Automatic Control, Vol. 11, Issue 2, pp. 161-167, 1966. 